\title{
Corrugated metal surface with pillars for terahertz surface plasmon polariton waveguide components
}

Ying Zhang, Yuehong Xu, Chunxiu Tian, Quan Xu, Xueqian Zhang, et al.

Ying Zhang, Yuehong Xu, Chunxiu Tian, Quan Xu, Xueqian Zhang, Yanfeng Li, Xixiang Zhang, Jiaguang Han, Weili Zhang, "Corrugated metal surface with pillars for terahertz surface plasmon polariton waveguide components," Proc. SPIE 10623, 2017 International Conference on Optical Instruments and Technology: IRMMW-THz Technologies and Applications, 1062302 (12 January 2018); doi: 10.1117/12.2295257

SPIE Event: International Conference on Optical Instruments and Technology 2017, 2017, Beijing, China 


\title{
Corrugated metal surface with pillars for terahertz surface plasmon polariton waveguide components
}

\author{
Ying Zhang ${ }^{\mathrm{a}}$, Yuehong Xu ${ }^{\mathrm{a}}$, Chunxiu Tian ${ }^{\mathrm{b}}$, Quan Xu${ }^{\mathrm{a}, \mathrm{b}}$, Xueqian Zhang ${ }^{\mathrm{a}}$,Yanfeng $\mathrm{Li}^{\mathrm{a}}$, Xixiang \\ Zhang $\dagger^{\mathrm{b}}$, Jiaguang Han*a, and Weili Zhang: ${ }^{\mathrm{a}, \mathrm{c}}$ \\ ${ }^{a}$ Center for Terahertz Waves and College of Precision Instrument and Optoelectronics Engineering, \\ Tianjin University, and the Key Laboratory of Optoelectronics Information and Technology Tianjin, \\ Ministry of Education of China, Tianjin, 300072, People's Republic of China \\ ${ }^{\mathrm{b}}$ Division of Physical Science and Engineering, King Abdullah University of Science and \\ Technology, Thuwal, 23955-6900, Saudi Arabia \\ ${ }^{\mathrm{c} S}$ School of Electrical and Computer Engineering, Oklahoma State University, Stillwater, Oklahoma \\ 74078, USA
}

\begin{abstract}
In the terahertz regime, due to perfect conductivity of most metals, it is hard to realize a strong confinement of Surface plasmon polaritons (SPPs) although a propagation loss could be sufficiently low. We experimentally demonstrated a structure with periodic pillars arranged on a thin metal surface that supports bound modes of spoof SPPs at terahertz (THz) frequencies. By using scanning near-field THz microscopy, the electric field distribution above the metal surface within a distance of $130 \mu \mathrm{m}$ was mapped. The results proved that this structure could guide spoof SPPs propagating along subwavelength waveguides, and at the same time reduce field expansion into free space. Further, for the development of integrated optical circuits, several components including straight waveguide, S-bend, Y-splitter and directional couplers were designed and characterized by the same method. We believe that the waveguide components proposed here will pave a new way for the development of flexible, wideband and compact photonic circuits operating at $\mathrm{THz}$ frequencies.
\end{abstract}

Keywords: Spoof surface plasmon polariton, waveguide component, corrugated metal surface.

\section{INTRODUCTION}

Surface Plasmon polaritons (SPPs) are electromagnetic waves propagating along metal-dielectric interfaces with exponential decay in the direction vertical to the interface. Recently, SPP based photonic circuits have attracted much attention because they possess both the capacity of information carrier of photonics and miniaturization of electronics, which open up a new way for next generation of integrated circuits, especially the information processing and transmission. In an effort to realize subwavelength waveguides and compact integrated circuits, it is vital to control the flow of SPPs. At terahertz (THz) frequencies, corrugated metal surface with slits, holes, or pillars have been employed to support spoof SPP modes, which have the same dispersion relation and tight confinements as SPPs in the optical region. Besides, it is also important to map the actual surface wave field on the metal surface directly.

\section{DISPERSION AND ANALYSIS OF SPOOF SPP MODE}

As the Fig. 1(a) shows, the waveguide structure that we chose was a row of periodic metal pillars arrayed on the metal surface. Previous study has demonstrated that the geometrical sizes of the metal structure have a significant influence on

† xixiang.zhang@kaust.edu.sa,

* jiaghan@tju.edu.cn

tweili.zhang@okstate.edu

2017 International Conference on Optical Instruments and Technology: IRMMW-THz Technologies and Their Applications, edited by Cunlin Zhang, Xi-Cheng Zhang, Zhiming Huang, Proc. of SPIE

Vol. 10623, 1062302 - (c) 2018 SPIE · CCC code: 0277-786X/18/\$18 · doi: 10.1117/12.2295257 
the dispersion relation of the SPP mode. For the consideration of good confinement, low loss and easy fabrication, the parameters were identified as follows: $w=200 \mu \mathrm{m}, p=100 \mu \mathrm{m}, l=50 \mu \mathrm{m}$, and $h=80 \mu \mathrm{m}$. Figure 1(b) shows the dispersion relation for the waveguide structure calculated using the eigen-mode solver of commercial software CST Microwave Studio. Within the first Brillouin zone, the $k_{\mathrm{x}}$ of the SPP mode became larger than the light line (black line) with increasing frequency. This means that the textured surface with pillars possesses a good field confinement ability. The inset of Fig. 1(b) displays a lateral profile of the normalized electric-field component $\left(E_{\mathrm{z}}\right)$ distribution of the SPP mode at $0.63 \mathrm{THz}$. It is apparent that the electric field was confined tightly on the surface of the pillar. In this frequency range, TM-like surface wave was supported and propagating on the surface of the metal structure. However, the group velocity of the SPP mode decreased to zero gradually at the first Brillouin zone boundary $\left(k_{\mathrm{x}}=\pi / p\right)$.

(a)

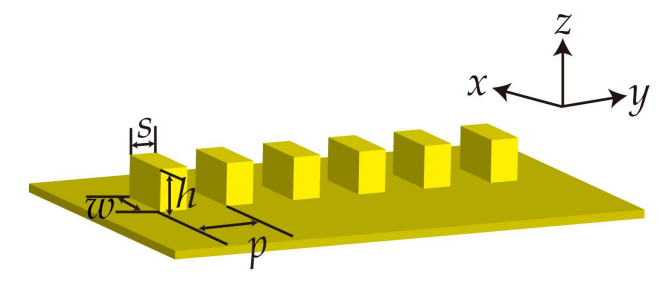

(b)

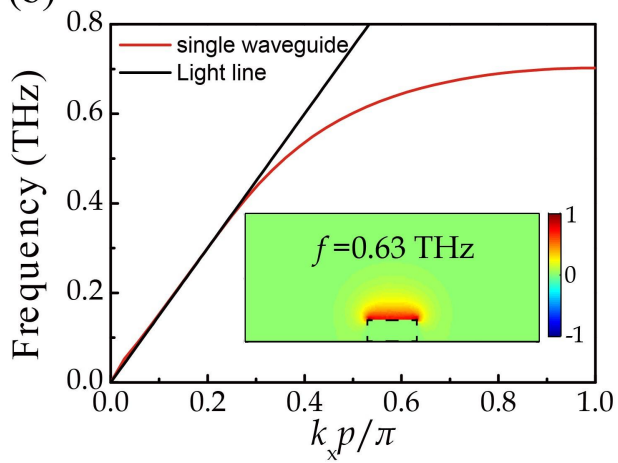

Figure. 1. Schematic of waveguide structure with geometrical parameters. (b) Dispersion relation of SPP mode for one row (red line) of metal pillars. The inset is normalized field magnitude of electric field component $\left(E_{\mathrm{z}}\right)$ for $y z$

air. cross-section of a single row waveguide at $0.63 \mathrm{THz}$. The dashed line indicates the boundary between metal and

\section{EXPERIMENT RESULTS}

In the present work, different waveguide components to be investigated included a straight waveguide, an S-bend, a Ysplitter and a directional coupler. The waveguide components were studied experimentally using a fiber-coupled scanning near-field $\mathrm{THz}$ microscopy system operating at $1.56 \mu \mathrm{m}$. Coupling of free-space THz radiation to the SPPs was achieved using periodic gratings.

\subsection{Straight waveguide}

Straight waveguides are fundamental elements for integrated optical circuits and as a result it is important to understand their propagation characteristics. Figure 2a shows a scanning electron microscopy (SEM) image of a part of the straight waveguide. The normalized power $\left(\left|E_{z}\right|^{2}\right)$ of the straight waveguide was imaged at $0.58 \mathrm{THz}$ (Figure $2 \mathrm{~b}$ ). The near-field image demonstrates well-confined SPPs guiding along the waveguide structure. Based on the field amplitude measured, the propagation length, horizontal confinement and field attenuation along the $z$ direction were quantitatively estimated. Figure 2c plots the measured amplitudes (blue dots) as a function of distance with an exponential fit (red line), which reveals a 1/e propagation length of $9.2 \mathrm{~mm}$. The corresponding propagation loss was about $8 \mathrm{~dB} / \mathrm{cm}$. Figure $2 \mathrm{~d}$ displays the normalized field amplitude as a function of $y$ coordinate at $x=3 \mathrm{~mm}$, which shows a full-width at halfmaximum of approximately $400 \mu \mathrm{m}$. The spatial evanescent extension (distance where the field drops to $1 / e$ of the surface value) in the air was $197 \mu \mathrm{m}$ obtained by exponential fit (solid line in Figure 2e). Compared with a bare metal surface, the spatial extension of the textured surface with pillars was reduced by several orders or magnitude. 
(a)

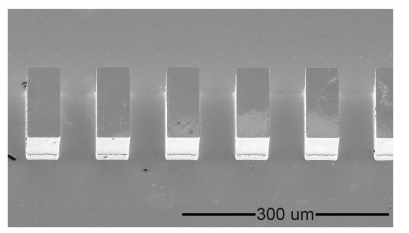

(b)

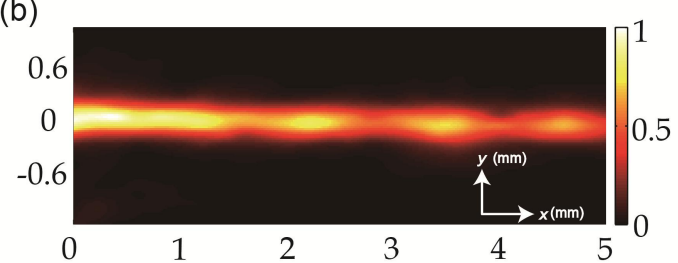

(c)

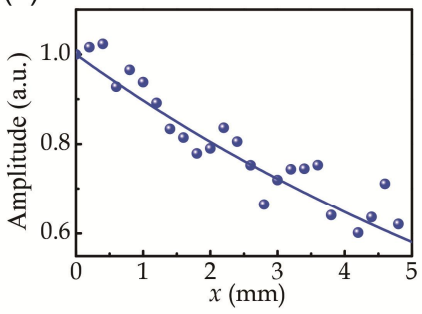

(d)

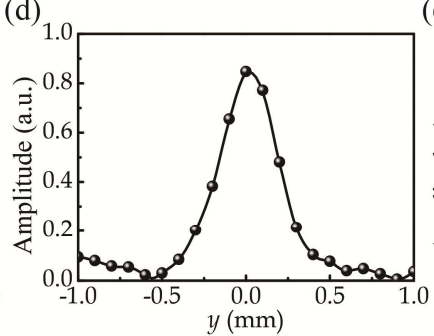

(e)

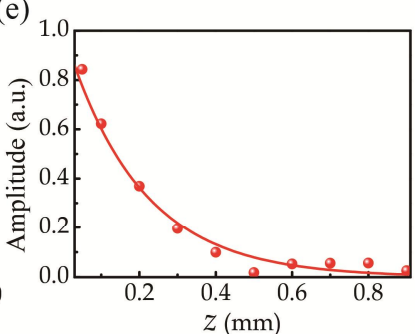

Figure 2. (a) SEM image of straight waveguide. (b) Near-field image of straight waveguide. The normalized power $\left|E_{z}\right|^{2}$ distribution at $0.58 \mathrm{THz}$ is shown. (c) Amplitude attenuation as a function of propagation distance. Blue dots represent experimental results and the solid line is exponential fit. (d) Measured electric field amplitude along line $x=3 \mathrm{~mm}$, showing the horizontal confinement. (e) Amplitude as function of increasing $z$. Red dots are experimental results and the solid line is exponential fit.

\subsection{S-bend, Y-splitter and directional coupler}

An S-bend waveguide based on cosine function was designed. The S-bend waveguide component was used as a junction to connect two straight waveguides offset with respect to each other. An S-bend waveguide component was also a basic element for a Y-splitter which was composed of two mirrored S-bends. Figure 3a shows a schematic of the S-bend and Y-splitter waveguides. There is a lower loss with increasing radius of curvature for a bend. With low loss taken into consideration, the S-bend with a radius of curvature of 911.8 um was fabricated and also applied in the Y-splitter. The Sseries waveguides were studied in a similar way by recording the electric field above the surface. For the S-bend waveguide, the normalized power at a frequency of $0.58 \mathrm{THz}$ is exhibited in Figure 3b, with a scanning area of $3.2 \times 4$ $\mathrm{mm}^{2}$. The result demonstrates well-confined SPPs guiding throughout the structure. The power attenuation was estimated to be $15 \mathrm{~dB} / \mathrm{cm}$ through the bend. Figure $3 \mathrm{c}$ delineates the normalized power distribution of the Y-splitter waveguide at $0.58 \mathrm{THz}$. At the junction, the two beams were separated successfully. At the end of the waveguide, the two arms were separated within a distance of $3 \mathrm{~mm}$. For a quantitative evaluation of the input and output powers, the normalized data along the lines $x=0 \mathrm{~mm}$ and $x=3 \mathrm{~mm}$ are shown in Figure $3 \mathrm{~d}$. The power in each branch was almost the same.Directional couplers (DCs) can be used as wavelength-selective components for filtering and separation of wavelengths, which are essential in the realization of demultiplexing components. The structure of a DC is displayed in Fig. 3(e). When two identical waveguide are close to one another, odd mode and even mode were supported by the entire structure. The dispersion relation of the two modes is different. This difference will lead to a phase difference. Over a distance of propagation, the accumulated phase difference reaches a value of $\pi$, and the mode power will be shifted completely from one waveguide to the other. The length corresponding to this shift is defined as the coupling length. Based on the numerical simulation, the coupling length is $2.83 \mathrm{~mm}$ when gap distance is $115 \mu \mathrm{m}$ at $0.6 \mathrm{THz}$. In the experiment, one of the straight waveguides was connected to the SPP source (the input in Figure 3e). The length of the coupling section was $L=2.8 \mathrm{~mm}$. The experimental results presented in Figure 3f show a good consistency with numerical calculation. 
(a)

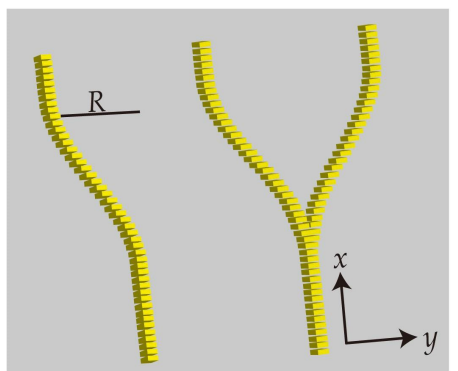

(c)

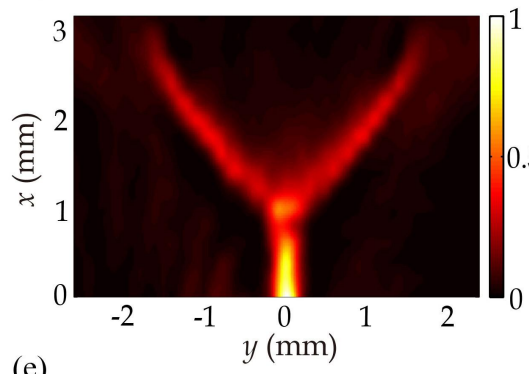

(e)

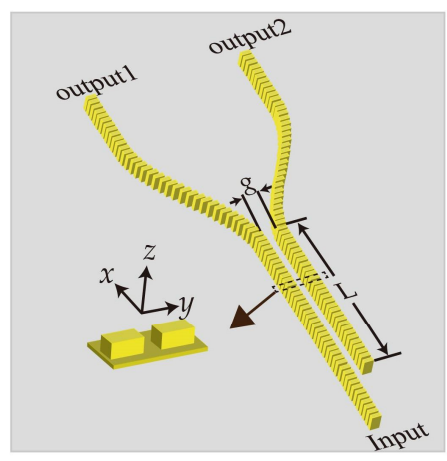

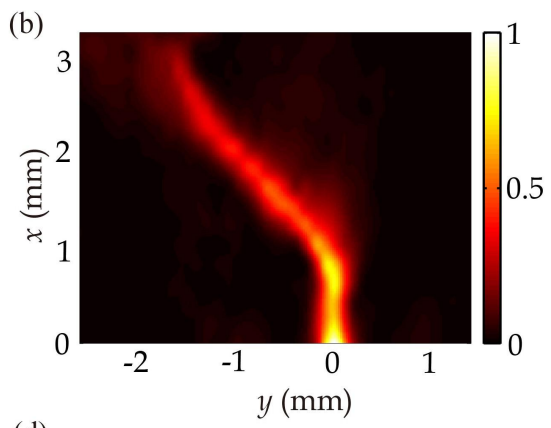

(d)

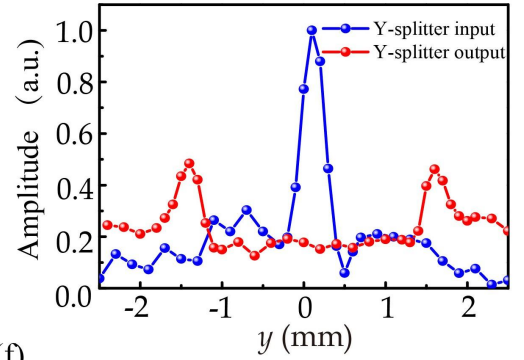

(f)

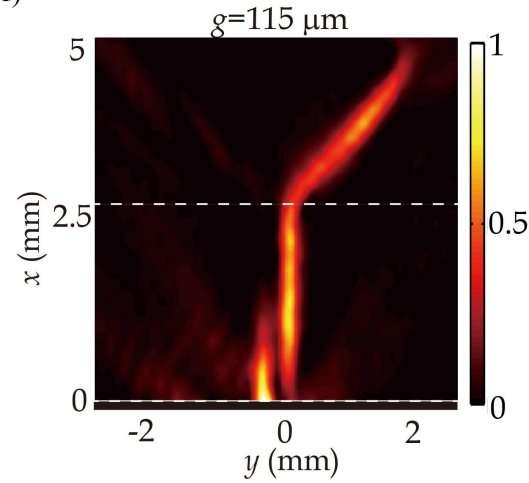

Figure 2. (a) Schematic of S-bend and Y-splitter waveguides. Near-field images corresponding to S-bend (b) and Y-splitter waveguides (c). The normalized power $\left|E_{z}\right|^{2}$ distribution at $0.58 \mathrm{THz}$ are shown. (d) Measured amplitude of electric field as a function of $y$ coordinate along the lines $x=0 \mathrm{~mm}$ (input) and $x=3 \mathrm{~mm}$ (output) of Ysplitter.(e) Schematic of DC with relevant parameter. (f) Normalized power distributions for DCs at $0.6 \mathrm{THz}$.

\section{REFERENCES}

[1] Pendry, J. B., Martín-Moreno, L., and Garcia-Vidal, F. J., “Mimicking Surface Plasmons with Structured Surfaces," Science 305 (5685), 847 (2004). [2] Williams, C. R., Andrews, S. R., Maier, S. A., Fernández-Domínguez, A. I., Martín-Moreno, L., and García-Vidal, F. J., "Highly confined guiding of terahertz surface plasmon polaritons on structured metal surfaces," Nature Photonics 2 (3), 175-179 (2008).

[3] Holmgaard, T., Chen, T., Bozhevolnyi, S. I., Markey, L., and Dereux, A. "Design and Characterization of Dielectric-Loaded Plasmonic Directional Couplers," Journal of Lightwave Technology 27 (24), 5521-5528 (2009). 